revista.economiaypolitica@ucuenca.edu.ec

Universidad de Cuenca

Ecuador

\title{
LEVANTAMIENTO DE PROCESOS COMO BASE PARA LA APLICACIÓN DE SISTEMAS DE COSTEO BASADO EN ACTIVIDADES EN EMPRESAS DE ENSAMBLAJE
}

Benavídez Vera, Erika X.; Segarra Farfán, Erika M.; Colina-Morles, Eliezer; Siguenza-Guzman, Lorena; Arcentales-Carrion, Rodrigo

LEVANTAMIENTO DE PROCESOS COMO BASE PARA LA APLICACIÓN DE SISTEMAS DE COSTEO

BASADO EN ACTIVIDADES EN EMPRESAS DE ENSAMBLAJE

Revista Economía y Política, vol. XV, núm. 30, 2019

Universidad de Cuenca, Ecuador

DOI: https://doi.org/10.25097/rep.n30.2019.03

(C) Universidad de Cuenca 2019

Esta obra está bajo una Licencia Creative Commons Atribución-NoComercial-Compartirlgual 4.0 Internacional. 


\section{LEVANTAMIENTO DE PROCESOS COMO BASE PARA LA APLICACION DE SISTEMAS DE COSTEO BASADO EN ACTIVIDADES EN EMPRESAS DE ENSAMBLAJE}

Process mapping as a basis for the application of activity-based costing systems in assembly industries

Erika X. Benavidez Vera

DOI: https://doi.org/10.25097/rep.n30.2019.03

Universidad de Cuenca, Ecuador

erika.benavidezv11@ucuenca.edu.ec

(iD http://orcid.org/0000-0003-2119-2040

Recepción: 03 Abril 2019

Erika M. Segarra Farfán

Aprobación: 24 Junio 2019

Universidad de Cuenca, Ecuador

erika.segarraf@ucuenca.edu.ec

(D) http://orcid.org/0000-0003-3509-5460

Eliezer Colina-Morles

Universidad de Cuenca, Ecuador

eliezer.colina@ucuenca.edu.ec

Lorena Siguenza-Guzman

Universidad de Cuenca, Ecuador

lorena.siguenza@ucuenca.edu.ec

(D) http://orcid.org/0000-0003-1367-5288

Rodrigo Arcentales-Carrion

Universidad de Cuenca, Ecuador

rodrigo.arcentales@ucuenca.edu.ec

(DD http://orcid.org/0000-0002-9700-8898

Recepción: 03 Abril 2019

Aprobación: 24 Junio 2019

\section{Resumen:}

Este trabajo propone el mapeo de procesos, como punto inicial, para la aplicación de un sistema de costos basado en actividades con evidencia reunida en dos líneas de ensamblaje, una manual y otra semiautomática. Para este propósito, se recopilan datos a través de técnicas de observación empírica y entrevistas individuales. Posteriormente, esta información se transfiere a hojas de proceso según las normas ISO 9001 y notación BPMN (Business Process Model and Notation) y se la analiza mediante técnicas estadísticas. Como resultado se pudo identificar y agrupar los procesos, subprocesos y actividades realizadas con los mismos recursos, logrando determinar el tiempo necesario para cada proceso de acuerdo a sus características, así como el número de repeticiones correspondiente a las actividades. Como conclusión, el trabajo permitió verificar que es posible realizar un análisis profundo de costos referentes a los procesos estratégicos, operacionales y de apoyo, por medio de un sistema de costeo basado en actividades, que permita analizar y precisar costos en base a los procesos críticos, buscando su optimización para un mejor desempeño de la organización.

Palabras ClaVE: Actividades, procesos, diagramas de flujo, ensamblaje, fichas de procesos, gestión, ISO, BPMN, TDABC.

\section{ABstract:}

This work proposes process mapping as a starting point for the application of a costing system based on activities, with evidence gathered in two assembly lines, one manual and the other semi-automated. For this purpose, data are collected through empirical observation techniques and individual interviews. Afterwards, this information is transferred to process sheets under ISO 9001 
standard and BPMN notation (Business Process Model and Notation) and analyzed by means of statistical techniques. As a result, it was possible to identify and group the processes, subprocesses and activities carried out with the same resources, achieving determining the time required for each process according to their characteristics, as well the number of repetitions corresponding to the activities. As a conclusion, this work allowed to verify that work that is it possible to accomplish a deep costs analysis related to strategic, operational and support processes, by mean of an activity-based system which permits analyzing and determining costs based upon critical processes, looking their optimization for a better performance of the organization.

KEYWORDS: Activities, processes, flowcharts, assembly, process cards, management, ISO, BPMN, TDABC.

\section{INTRODUCCIÓN}

El ensamblaje de productos representa un importante sector del quehacer industrial, por lo que, las empresas buscan obtener una mayor participación en el mercado al contar con factores diferenciadores. Lamentablemente, a causa de cambios en el comercio internacional, el avance tecnológico y su aplicación en el desarrollo de nuevos mercados, una gran cantidad de empresas industriales han volcado su atención al ensamblaje de productos con una planificación bajo demanda. Para esto, dichas organizaciones han buscado mejorar su rentabilidad y capacidad de producción, optimizando sus actividades y recursos, con el fin de obtener resultados favorables y productos de calidad, que les permitan desarrollar actividades dentro del entorno cambiante y competitivo en el que se desarrollan.

Sin embargo, esta situación está envuelta en un ambiente complejo, donde las empresas pueden ser perjudicadas en su desempeño y sus costos, por factores externos como medidas gubernamentales y la competencia; y por factores internos, como el cambio de personal tanto operativo como de dirección, políticas empresariales nuevas, entre otros. Dados los factores mencionados es necesario que el sector de las ensambladoras incorpore nuevos modelos y métodos asociados a la gestión en busca de obtener mejores resultados, incrementando su competitividad y rentabilidad a través de la optimización de los costos de operación.

Bajo este contexto, este trabajo se enfoca en el análisis realizado a la gestión de dos empresas de ensamblaje, una de producción manual de motocicletas y otra de producción semiautomatizada de tarjetas electrónicas, previo a la implementación de un modelo para la optimización de sus procesos y costos. Así, el principal objetivo es impulsar el desarrollo de un modelo de gestión de procesos por medio de la identificación de procesos estratégicos, de apoyo y operacionales en los casos estudiados; así como también, la evaluación de un sistema que se acople a la técnica de gestión de costos, conocida como Costeo Basado en el Tiempo invertido por Actividad (TDABC por sus siglas en inglés, Time Driven Activity Based Costing).

\section{REVISión DE LA LiTERATURA}

Un proceso es un conjunto de actividades, paralelas o no, que siguen una secuencia y son realizadas por una persona sobre un insumo, al cual le agrega valor para luego suministrar un producto a un cliente (Agudelo Tobón \& Escobar Bolívar, 2008). Con este enfoque, Oakland (1989) completa este concepto como la transformación de una serie de entradas, tales como, operaciones y métodos, en salidas que buscan satisfacer las necesidades y expectativas de los consumidores en forma de productos, servicios, información, o en general, resultados.

Todas las operaciones que realizan las empresas constituyen procesos en los cuales existe participación de personas en base a las actividades que ejecutan. Los procesos descritos se clasifican en estratégicos, operativos y de apoyo. Los primeros están relacionados con la estrategia de la organización. Los operativos se enfocan en la misión del negocio y satisfacen necesidades específicas de los clientes; además, son los que generan valor agregado y por los cuales el cliente está dispuesto a pagar un monto. Finalmente, los de apoyo son los que 
proporcionan los medios y soporte necesarios para que los procesos operativos puedan ser ejecutados (Bravo Carrasco, 2009, p. 30).

La manera más clara de representar gráficamente la estructura de los procesos es mediante un mapa. Para la elaboración y fácil interpretación del mismo, es indispensable un análisis previo de las posibles agrupaciones en las que se pueda colocar los procesos identificados (Beltrán Sanz, Carmona Calvo, Carrasco Pérez, Rivas Zapata, \& Tejedor Panchon, 2009). Dichas uniones se pueden concebir como macro-procesos que incorporan dentro de sí otros procesos y a su vez, estos se puedan desplegar en subprocesos. Esto facilita la interrelación e interpretación de todo el mapa, estableciendo afinidades.

La descripción de un proceso se debe centrar tanto en las actividades como en el control de las mismas, buscando que estas se realicen de una manera eficaz. Una herramienta fundamental para dicha descripción es la ficha de proceso, que representa un respaldo de información para recabar características relevantes para el control de las actividades definidas en el diagrama y la gestión del proceso (Beltrán Sanz et al., 2009).

Posterior a la aplicación de las fichas, los diagramas de flujo son el primer nivel de información del proceso y constituyen una herramienta para que el equipo de trabajo llegue a un consenso sobre los diferentes elementos del flujo. Dentro de un diagrama de flujo es necesario tener símbolos que proporcionen un significado preciso y claro al momento de utilizarlos. Internacionalmente, varias instituciones han empleado diferentes símbolos de diagramación, pero los más importantes y generalmente utilizados son los establecidos por la Organización Internacional de Estandarización, ISO (Franklin Fincowsky, 2009).

Según la norma ISO 9000, la gestión es un conjunto de "actividades coordinadas para dirigir y controlar una organización” (International Organization for Standardization, 2015). Así, una vez definidos los conceptos de proceso y gestión, se determina que la gestión por procesos es una manera sistémica de identificar, comprender y acrecentar el valor agregado de todos los procesos de la empresa para cumplir con la estrategia de la organización y aumentar el nivel de satisfacción de los clientes (Bravo Carrasco, 2009). Esta visión sistémica colabora con el incremento de la productividad y control de gestión para mejorar variables clave, como tiempo, calidad y costo.

Dados los entornos y mercados competitivos y dinámicos, a las empresas les resulta esencial gestionar actividades y recursos, adoptando instrumentos que les permitan establecer sus sistemas de gestión (Beltrán Sanz et al., 2009). Existen varios modelos de gestión por procesos que pueden ser aplicados, como la Gestión de Procesos basada en la Norma ISO 9001 que promulga la adopción de un enfoque basado en procesos; en un sistema de gestión de la calidad para aumentar la satisfacción del cliente mediante el cumplimiento de sus requisitos (Beltrán Sanz, et al., 2009, p. 21). Por su parte, BPM (Business Process Management) hace referencia a diferentes métodos, herramientas y tecnologías utilizados para el diseño, representación, análisis y control de los procesos de negocio operacionales (Hitpass, 2017).

Para la obtención de información necesaria se propone una combinación de los dos modelos de gestión mencionados anteriormente, siendo necesario estructurar una metodología de levantamiento de procesos. Para plantear la misma, se estudió literatura científica referente al tema, considerando: la Guía de levantamiento de procesos (Ortega Vindas, 2009), el Manual de gestión de procesos (Servicio de Evaluación, Planificación y Calidad, 2011), la Guía para una gestión basada en procesos (Beltrán Sanz et al., 2009) y la Guía para el levantamiento de procesos institucionales y diseño de indicadores (Área de Planificación y Evaluación MOPT Seguridad Vial, 2015). Las cuatro guías mencionadas tienen como sustento para su elaboración la Norma ISO 9000 (International Organization for Standardization, 2000a), el Servicio de Calidad de la Atención Sanitaria (Servicio de Salud de Castilla-La Mancha, 2002), el Instituto Centroamericano de Administración Pública - ICAP (2006), el Modelo de Excelencia EFQM (European Foundation for Quality Management, 1988), la Norma ISO 9001 (International Organization for Standardization, 2000b), el Plan Pegasus (Universidad Politécnica de Valencia, 2006), la UNE 93200 (Asociación Española de Normalización, 2008), el Cuadro de Mando Integral (Kaplan \& Norton, 1997), Estrategia Competitiva (Porter, 2000), la Norma técnica Inteco (Instituto de Normas Técnicas de Costa 
Rica, 2004), entre otras. Así, la literatura revisada posee un sustento y base sólida que permite sugerir que la metodología propuesta, con base a aspectos comunes de las previamente analizadas, es adecuada y necesaria para los casos estudiados. La misma está constituida por las siguientes etapas fundamentales: planificación de los manuales de procesos, conocimiento de la filosofía corporativa e identificación y descripción de los procesos (Tabla 1 ).

TABLA 1.

Metodología para el levantamiento de procesos

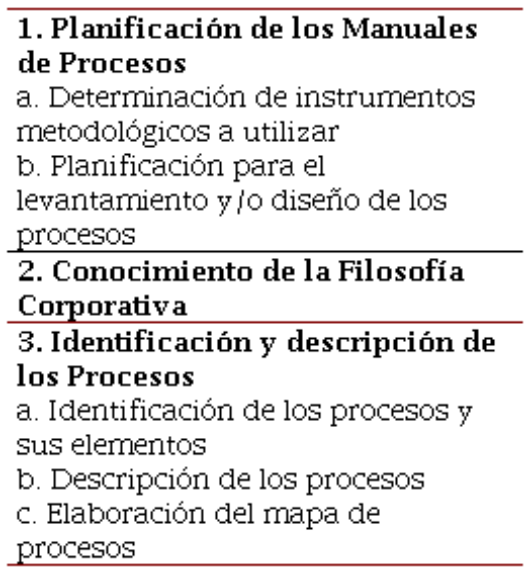

Elaboración propia con datos de Ortega Vindas (2009), Servicio de Evaluación, Planificación y Calidad (2011), Beltrán Sanz, Carmona Calvo, Carrasco Pérez, Rivas Zapata, \& Tejedor Panchon (2009), Área de Planificación y Evaluación MOPT Seguridad Vial (2015)

La aplicación de esta metodología permitirá la realización de análisis posteriores, principalmente en relación con la aplicación del sistema de costeo TDABC desarrollada por Kaplan y Anderson (2007), el cual facilita a las organizaciones el manejo de costos de recursos utilizados por actividad para la producción de un bien o servicio y al mismo tiempo, conocer el consumo de cada área, el tiempo de duración, costo de cada actividad y el costo total de operación. En este sentido, TDABC representa una técnica de gestión de costos de los procesos, que permite desarrollar información precisa sobre los mismos en una amplia gama de actividades (Beltrán Sanz et al., 2009). En este modelo los costos se asignan directamente a su objeto de estudio en base a dos parámetros: el costo por unidad de tiempo de la capacidad suministrada de recursos y el tiempo estimado que requiere cada actividad (Kaplan \& Anderson, 2007). Por ello, se considera como primera etapa, la identificación de actividades que son realizadas con los mismos medios para constituir los "grupos de recursos" y la determinación del tiempo incurrido para cada tarea de acuerdo a sus características (Arbulo Lopez \& Fortuny-Santos, 2011). Dadas esas ventajas que presenta TDABC, el levantamiento de procesos, actividades y tiempos constituyen una base fundamental para la metodología de cálculo final del costo de bienes y/o servicios según este sistema.

Dentro de este contexto, en el mercado ecuatoriano se han realizado estudios relacionados al tema de esta investigación. Uno de ellos es la propuesta de un modelo de gestión por procesos en la ensambladora de motocicletas CKD (Completely Knock Down) de la empresa Vycast Cía. Ltda., bajo lineamientos de ISO 9001 (Astudillo Delgado \& González Martínez, 2014). Este estudio establece que es posible medir la eficiencia y eficacia de las operaciones al equilibrar variables que intervienen en el proceso de producción, como: personas, procesos, calidad, tiempo, clientes, entre otros factores, todos indispensables para el cumplimiento de los objetivos de la empresa.

Otra investigación estudia el plan de negocio para la creación del área de ensamblaje aplicado a la Empresa de Televisores Ensab, para la comercialización a nivel nacional (Orbe Aguirre, 2018). El autor establece que 
es posible reactivar el sector productivo del país mediante un efectivo proceso de ensamblaje y controles de calidad rigurosos, con el fin de optimizar procesos y reducir costos.

Finalmente, se encuentra el estudio realizado con relación a la producción nacional de bicicletas eléctricas con componentes nacionales (Criollo Coronado, 2016). Este trabajo se centra en un análisis financiero y de procesos que concluye con un proyecto generador de utilidades económicamente rentable, el cual contribuye en el cambio de la matriz productiva del país con una mayor productividad y una mejora de la calidad, sustituyendo importaciones y considerando la innovación y tecnología como factores fundamentales dentro del proceso productivo.

Adicionalmente, en otras publicaciones relacionadas como "Modelo de Optimización para el cálculo de tiempos estándar en procesos de ensamblaje” (Guerrero, Guamán, Colina Morles, \& Siguenza-Guzmán, 2019), "Predicción de tiempos estándar en líneas de ensamble usando mínimos cuadrados en modelos lineales multivariables" (Ramírez \& Guamán, 2019) y "Modelo matemático basado en programación lineal y lógica difusa para predicción de tiempos en industrias de ensamble de bicicletas” (Rodas D., Colina Morles, Peña, \& Siguenza-Guzmán, 2019); se concluye que gracias a un adecuado levantamiento de procesos y tiempos, es posible construir modelos matemáticos que sirven como herramientas en el área de ingeniería de métodos para el cálculo de tiempos estándar.

En base a las investigaciones realizadas se determina la construcción de modelos genéricos de procesos para empresas de ensamblaje (Luzuriaga, 2019), aplicables en el contexto de líneas de producción de otras empresas afines, facilitando la estandarización de procesos tanto administrativos como de ensamble, mediante la utilización de herramientas como BPM (Business Process Management).

\section{Materiales y MÉtodos}

El trabajo fue desarrollado en dos áreas: operativa y administrativa, de una empresa de producción manual de motocicletas y otra de producción semiautomatizada de tarjetas electrónicas. Por razones de confidencialidad, se mantiene bajo privacidad tanto los nombres reales de las organizaciones, como de sus líneas de productos.

Las compañías estudiadas producen una diversidad de modelos ensamblados; en el caso de las motocicletas se tienen diez modelos: XI... X10 y en el caso de tarjetas electrónicas se consideran 18 modelos: A1... A12 / B1 - C1 / B2 - C2 / B3 - C3 / B4 - C4 / B5 - C5 / D1 / D2 y dos modelos de teclados A13 y A14.

El trabajo requirió el levantamiento de todos los procesos de las empresas casos de estudio, para luego realizar un análisis riguroso de resultados y hallazgos. El enfoque metodológico fue de tipo mixto, cuantitativo y cualitativo.

Se realizó un análisis cuantitativo, dada la necesidad de determinar con valores numéricos los procesos trascendentes o críticos dentro de las empresas, número de actividades, número de personas y tiempos, utilizando técnicas como el cronometraje. El análisis cualitativo llevado a cabo consistió en conocer a fondo la cultura organizacional (costumbres, situaciones, comportamientos) de las empresas para entender los procesos levantados y su importancia para el personal, por medio de técnicas como la observación y la aplicación de entrevistas. Este análisis culminó en la validación de los resultados obtenidos y el esclarecimiento de hallazgos inesperados y/o posibles contradicciones (Tabla 2). 
TABLA 2.

Metodología para el levantamiento y análisis de procesos

\begin{tabular}{|c|c|c|}
\hline \multirow{4}{*}{$\begin{array}{l}\text { Levantamiento de } \\
\text { procesos }\end{array}$} & \multicolumn{2}{|c|}{ Recolección de información de la estructura organizacional del objeto de estudio } \\
\hline & \multicolumn{2}{|c|}{ Preparación y socialización con el objeto de estudio } \\
\hline & \multirow[t]{2}{*}{ Levantamiento de procesos } & Procesos administrativos: entrevistas \\
\hline & & $\begin{array}{l}\text { Procesos de ensamblaje: toma de tiempos } \\
\text { con cronómetro }\end{array}$ \\
\hline \multirow[t]{2}{*}{ Análisis de procesos } & $\begin{array}{l}\text { Procesamiento y clasificación de la } \\
\text { información }\end{array}$ & Fichas basadas ISO 9001 \\
\hline & Diagramación de los procesos & Bizagi Modeler \\
\hline
\end{tabular}

Los autores

\subsection{Levantamiento de procesos}

Para proceder con la identificación y levantamiento de procesos del área administrativa se recurrió a entrevistas semiestructuradas y no estructuradas para la recolección de datos, lo que permitió tener un mayor grado de flexibilidad y ajuste con los entrevistados. La ficha de procesos basada en BPM e ISO 9001-2015, junto con una grabadora digital, fueron utilizadas como instrumentos para recolectar los principales componentes de las actividades. En el área operativa se utilizó la técnica de observación como principal método de recolección de datos. La metodología de toma de tiempos seleccionada fue la de General Electric, que recomienda cierta cantidad de ciclos en base al tiempo que tome la ejecución de las actividades que pertenecen a un mismo ciclo (García Criollo, 2005, p. 212).

Una vez levantados los procesos por medio de entrevista y observación, se procede con la documentación de los mismos en las fichas mencionadas, para continuar con la validación y aprobación con cada una de las personas responsables.

\subsection{Análisis de procesos}

Para el análisis e interpretación de la información plasmada en las fichas de procesos, se construyeron diagramas de flujos usando la versión gratuita de la plataforma Bizagi Modeler [1], que se basa en la notación BPMN.

Una vez determinados todos los elementos necesarios para la elaboración de los flujogramas por empresa, se procede con la diagramación de cada una de las actividades descritas en las fichas. Esto con el fin de posteriormente poder relacionar todos los subprocesos de los departamentos y elaborar un macro-proceso, que permita comprender de mejor manera el funcionamiento de cada organización.

Con toda la información recolectada y procesada, se elaboran mapas en los que se establecen los macroprocesos de la cadena de valor de las empresas y se realiza una categorización en estratégicos, operacionales y de apoyo. Esto permite identificar los procesos y actividades críticas de cada una de las empresas y realizar un análisis estadístico usando Diagramas de Caja, por medio del lenguaje de programación para análisis estadístico denominado R Project. Finalmente, el análisis permite, de acuerdo a la metodología para el levantamiento y análisis de procesos realizada en primera instancia, conocer por completo los procesos y 
actividades de los casos de estudio, previo al cálculo de costes a través de TDABC; la cual, en su primer paso propone identificar los diversos grupos de recursos (departamentos o procesos).

Posterior al análisis; se realizan los levantamientos y el mapa, así como el modelado de los mismos, contribuyendo con estos insumos al cálculo de costes.

\section{Resultados}

A continuación, se presentan los principales hallazgos obtenidos en el desarrollo del presente trabajo.

\subsection{Levantamiento de procesos}

Una vez levantada la información se procede a realizar los respectivos análisis que contribuyen en el desarrollo de un modelo de gestión por procesos mediante la identificación de procesos estratégicos, de apoyo y operativos, y establece una base para la aplicación del sistema de costeo TDABC, cumpliendo así con el objetivo principal de esta investigación. Para la empresa de producción manual de motocicletas, se identificaron 382 subprocesos, conformados por un total de 1763 actividades; mientras que para la empresa de producción semiautomatizada de tarjetas electrónicas, se identificó un total de 218 subprocesos, constituidos por 965 actividades.

Levantados todos los datos de procesos, en las fichas desarrollada bajo BPM e ISO, se obtuvo información importante relativa a cada proceso, subprocesos, y actividades; así como, el tiempo promedio de cada uno de ellos. De igual forma, se obtuvo los condicionales existentes en el levantamiento de cada actividad, así como los recursos humanos $(\mathrm{RH})$, recursos tecnológicos $(\mathrm{RT})$ y recursos materiales $(\mathrm{RM})$ utilizados en las mismas.

Finalmente, se pudo determinar el número de repeticiones que conlleva desarrollar cada una de las actividades, dentro de cada proceso o subproceso, estos datos son considerados el principal insumo, al cual se hacía referencia en la literatura para la aplicación del primer paso del sistema de costes TDABC.

A manera de ejemplo, tanto en la Tabla 3, como en el Gráfico 1, se puede visualizar el proceso de ensamblaje correspondiente a teclados (tarjetas electrónicas).

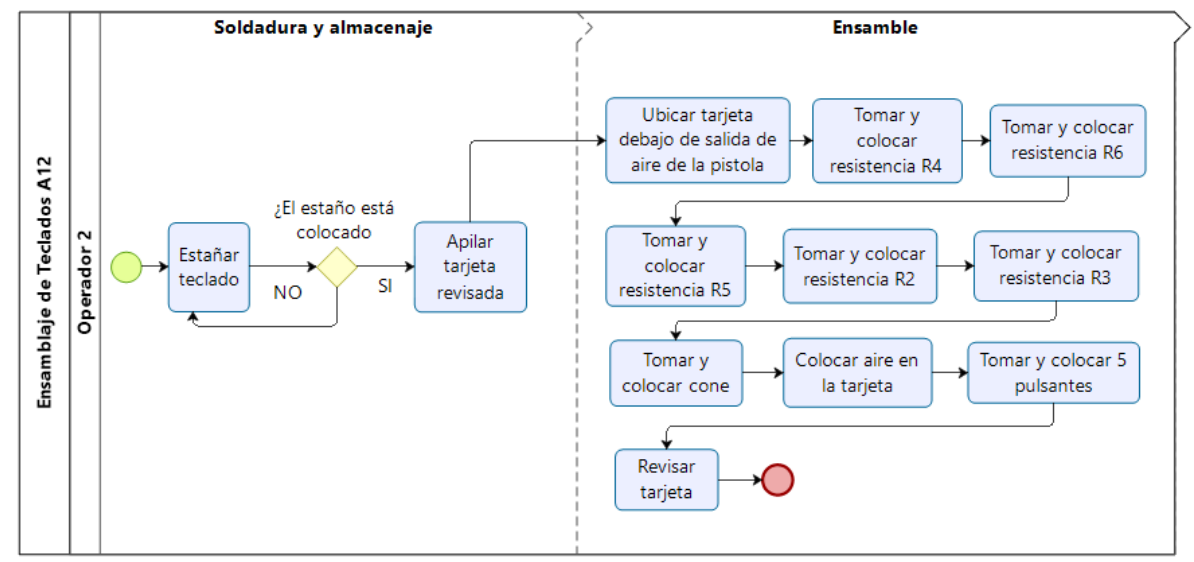

GRÁFICO 1.

Diagrama de flujo de proceso de ensamblaje de teclados A12

Elaboración propia a partir de las fichas de levantamiento de procesos para la empresa de producción semiautomatizada de tarjetas electrónicas 
Erika X. Benavídez Vera, et al. LEVANTAMiento de Procesos COMO BaSE PARA LA APLICACIÓN DE SISTEMAS...

TABLA 3.

Proceso de ensamblaje de teclado A13

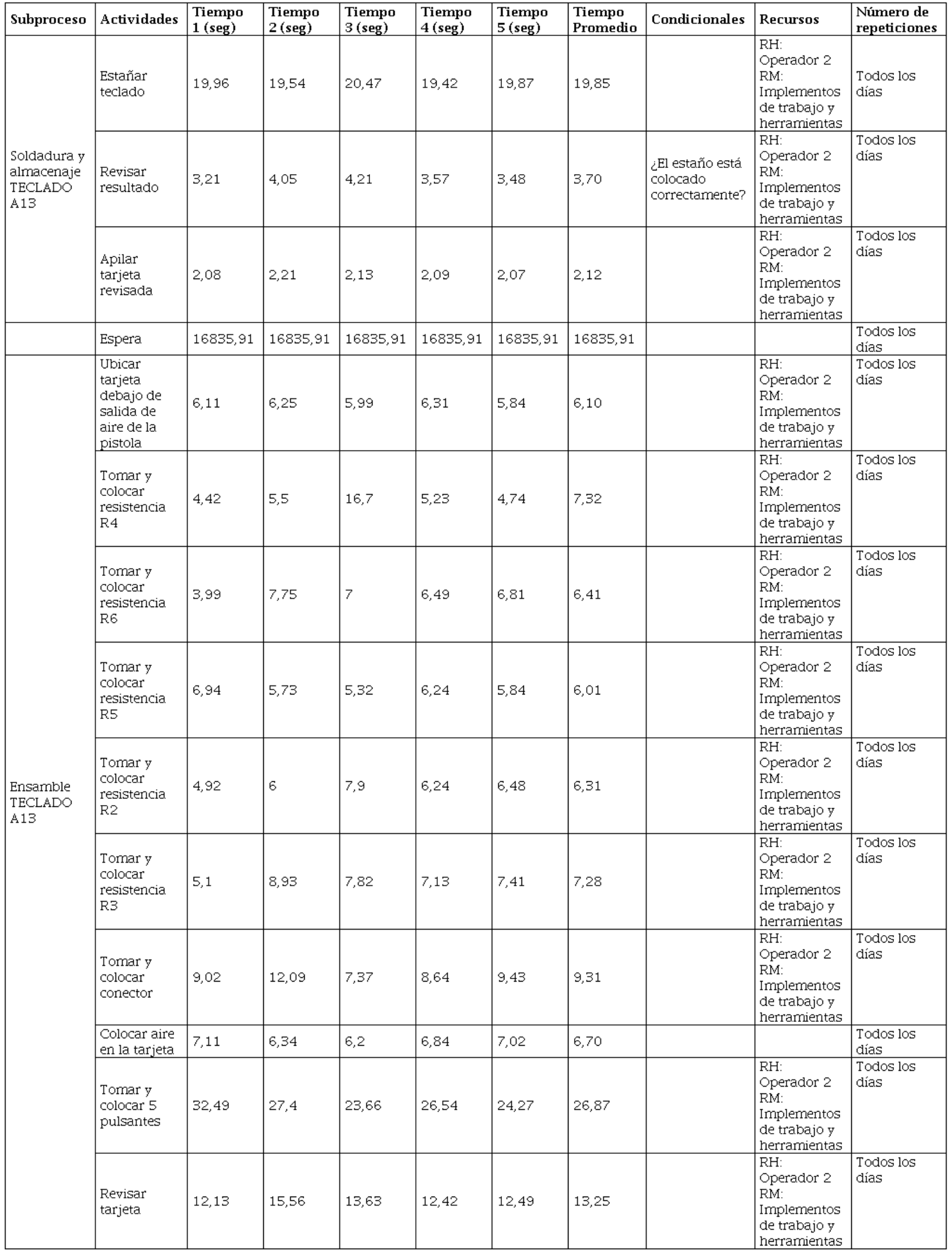

Elaboración propia a partir de las fichas de levantamiento de procesos para la empresa de producción semiautomatizada de tarjetas electrónicas 


\subsection{Análisis de procesos}

En base al levantamiento de procesos ejecutado en las empresas, en el Gráfico 2 y Gráfico 3 se presentan ejemplos de los diagramas elaborados por medio de la plataforma de Bizagi:

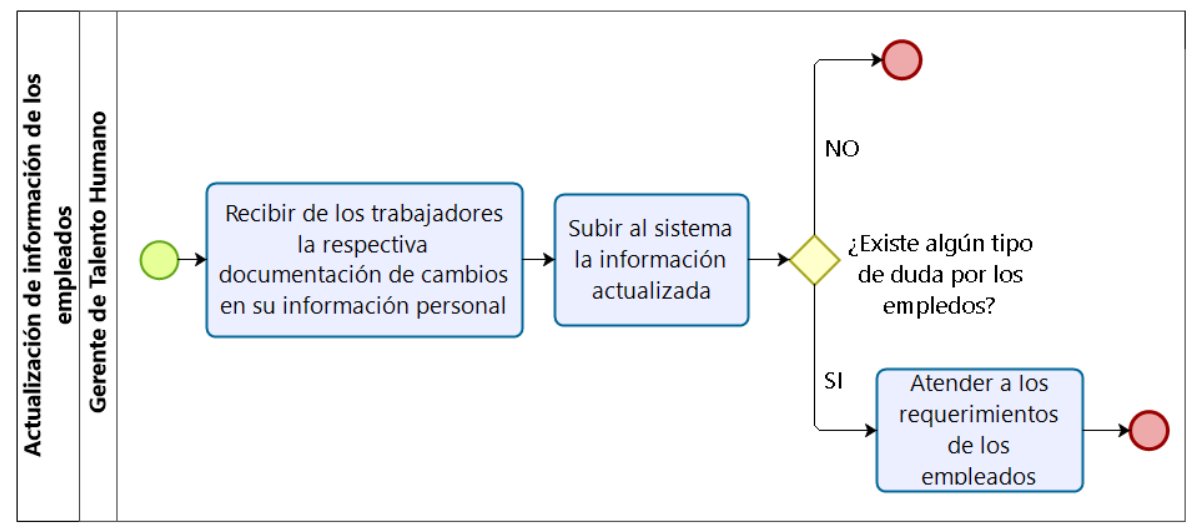

\section{GRÁFICO 2.}

Diagrama de flujo de actualización de información de los empleados realizado en el Departamento de Talento Humano

Elaboración propia a partir de las fichas de levantamiento de procesos para la empresa de producción semiautomatizada de tarjetas electrónicas y para la empresa de producción manual de motocicletas.

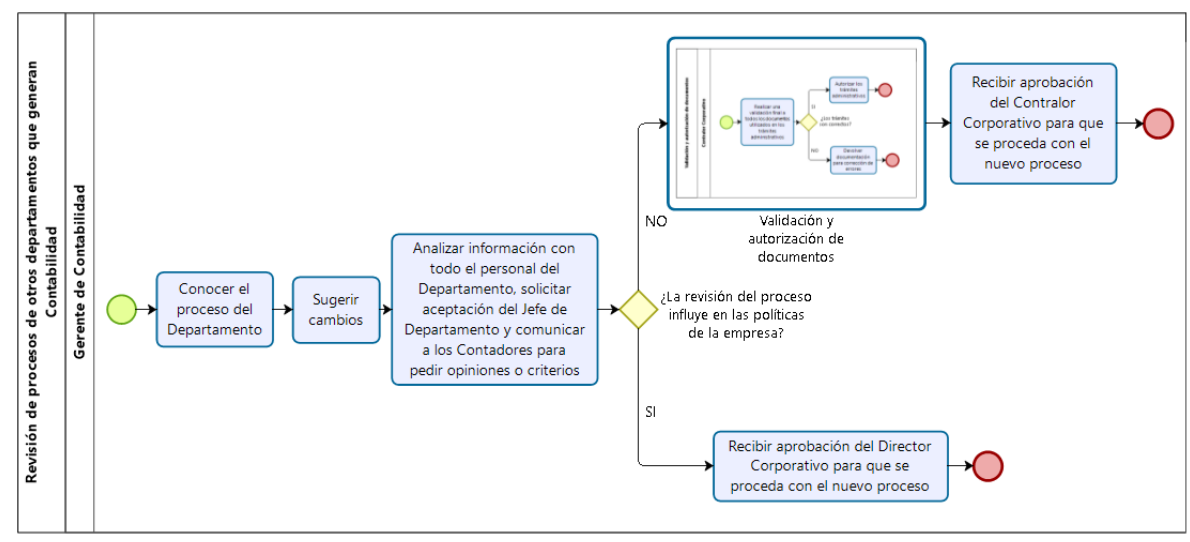

GRÁFICO 3.

Diagrama de flujo de revisión de procesos de otros departamentos que generan contabilidad realizado en el Departamento de Contabilidad

Elaboración propia a partir de las fichas de levantamiento de procesos para la empresa de producción semiautomatizada de tarjetas electrónicas y para la empresa de producción manual de motocicletas.

Para la construcción del mapa de procesos se estableció una lista de macro-procesos en base a la información levantada y se realizó la categorización de los mismos en estratégicos, operacionales y de apoyo. Seguidamente, se procedió con su elaboración, que a nivel macro, es similar para los dos casos de estudio; pero, a nivel de procesos, las actividades varían considerablemente para cada organización. El Gráfico 4 ilustra el mapa para las empresas estudiadas. 


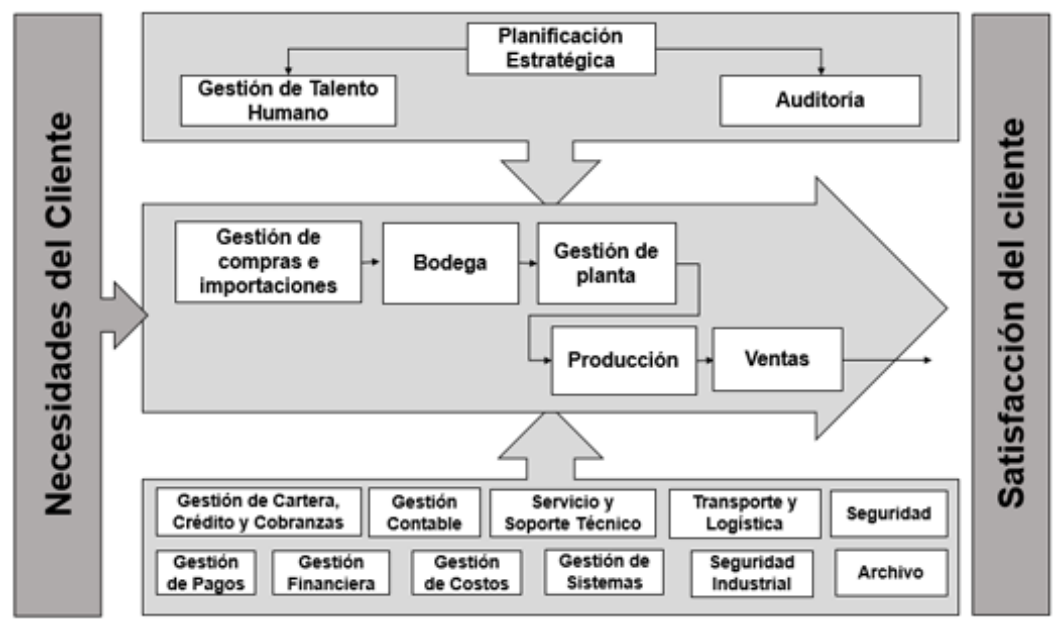

GRÁFICO 4.

Mapa de procesos de la empresa de producción semiautomatizada de tarjetas electrónicas y de la empresa de producción manual de motocicletas

Elaboración propia a partir de las fichas y diagramas de levantamiento de procesos para la empresa de producción semiautomatizada de tarjetas electrónicas y para la empresa de producción manual de motocicletas.

\subsection{Análisis de procesos críticos}

Para realizar el análisis estadístico de los procesos críticos de las empresas se determinó la cadena de valor conformada por la gestión de compras e importaciones, bodega, planta, producción y ventas, como se muestra en el Gráfico 5.

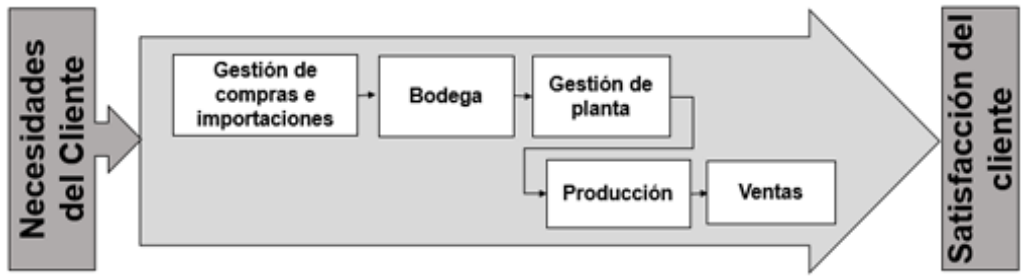

GRÁFICO 5.

Cadena de valor de la empresa de producción semiautomatizada de tarjetas electrónicas y de la empresa de producción manual de motocicletas.

Elaboración propia a partir de las fichas y diagramas de levantamiento de procesos para la empresa de producción semiautomatizada de tarjetas electrónicas y para la empresa de producción manual de motocicletas.

Para proceder con el análisis estadístico se utilizó una base de datos que contiene todos los macro-procesos operativos de la cadena de valor y los tiempos que toma realizar cada uno de estos en las dos empresas estudiadas. Con ello, se pudo determinar que en dichas organizaciones el macro-proceso de gestión de producción es el que conlleva mayor tiempo en su ejecución. Por tal razón, los análisis posteriores (Diagrama de Pareto, Diagrama de Cajas o Boxplot e Histograma) se enfocan en este macro-proceso.

\subsubsection{Diagrama de Pareto}

Con respecto a la empresa de producción semiautomatizada de tarjetas electrónicas, dentro del proceso de ensamble se logró determinar que el ensamblaje de modelos automáticos A2 y A3 constituyen los subprocesos críticos, debido a que conllevan un mayor tiempo de ejecución. 
Para la construcción del diagrama de Pareto se consideraron el tiempo de ejecución, porcentaje acumulado y tiempo acumulado de las actividades indicadas en la Tabla 4 y Gráfico 6. Se determinó que el mayor tiempo de ocupación son las "esperas" entre ciertas actividades que tienen las tarjetas electrónicas ensambladas, puesto que estas se mantienen almacenadas por un tiempo considerable.

TABLA 4.

Análisis estadístico del proceso de ensamblaje de los modelos de tarjetas electrónicas A10 y A11 Android

\begin{tabular}{|c|c|c|c|}
\hline \multicolumn{4}{|c|}{ Modelo de tarjetas electrónicas A2 y A3 Android } \\
\hline Actividades & Tiempo & $\begin{array}{l}\% \\
\text { Acumulado }\end{array}$ & $\begin{array}{l}\text { Tiempo } \\
\text { Acumulado }\end{array}$ \\
\hline Espera & 26855,00 & $81,18 \%$ & 26855,00 \\
\hline $\begin{array}{l}\text { Espera } \\
\text { almacenado }\end{array}$ & 5880,00 & $98,95 \%$ & 32735,00 \\
\hline $\begin{array}{l}\text { Ensamblar lado } \\
\text { top }\end{array}$ & 120,00 & $99,31 \%$ & 32855,00 \\
\hline Probar tarjeta & 55,36 & $99,48 \%$ & 32910,36 \\
\hline $\begin{array}{l}\text { Grabar } \\
\text { software }\end{array}$ & 48,78 & $99,63 \%$ & 32959,14 \\
\hline $\begin{array}{l}\text { Ensamblar lado } \\
\text { bottom }\end{array}$ & 45,00 & $99,76 \%$ & 33004,14 \\
\hline $\begin{array}{l}\text { Colocar } \\
\text { protector } \\
\text { plástico y } \\
\text { ubicarlas en el } \\
\text { cartón }\end{array}$ & 13,73 & $99,81 \%$ & 33017,87 \\
\hline $\begin{array}{l}\text { Sacar } \\
\text { protecciones de } \\
\text { tarjeta }\end{array}$ & 12,28 & $99,84 \%$ & 33030,15 \\
\hline Almacenar & 12,00 & $99,88 \%$ & 33042,15 \\
\hline $\begin{array}{l}\text { Inspección } \\
\text { visual y colocar } \\
\text { tarjeta }\end{array}$ & 11,93 & $99,92 \%$ & 33054,07 \\
\hline Sacar tarjeta & 6,39 & $99,93 \%$ & 33060,46 \\
\hline $\begin{array}{l}\text { Sacar de rack y } \\
\text { cortar tarjeta }\end{array}$ & 6,31 & $99,95 \%$ & 33066,77 \\
\hline $\begin{array}{l}\text { Colocar } \\
\text { disipador }\end{array}$ & 6,08 & $99,97 \%$ & 33072,85 \\
\hline Colocar sello & 5,37 & $99,99 \%$ & 33078,22 \\
\hline Marcar tarjeta & 3,82 & $100,00 \%$ & 33082,04 \\
\hline
\end{tabular}

Elaboración propia a partir de las fichas levantamiento de procesos para la empresa de producción semiautomatizada de tarjetas electrónicas. 


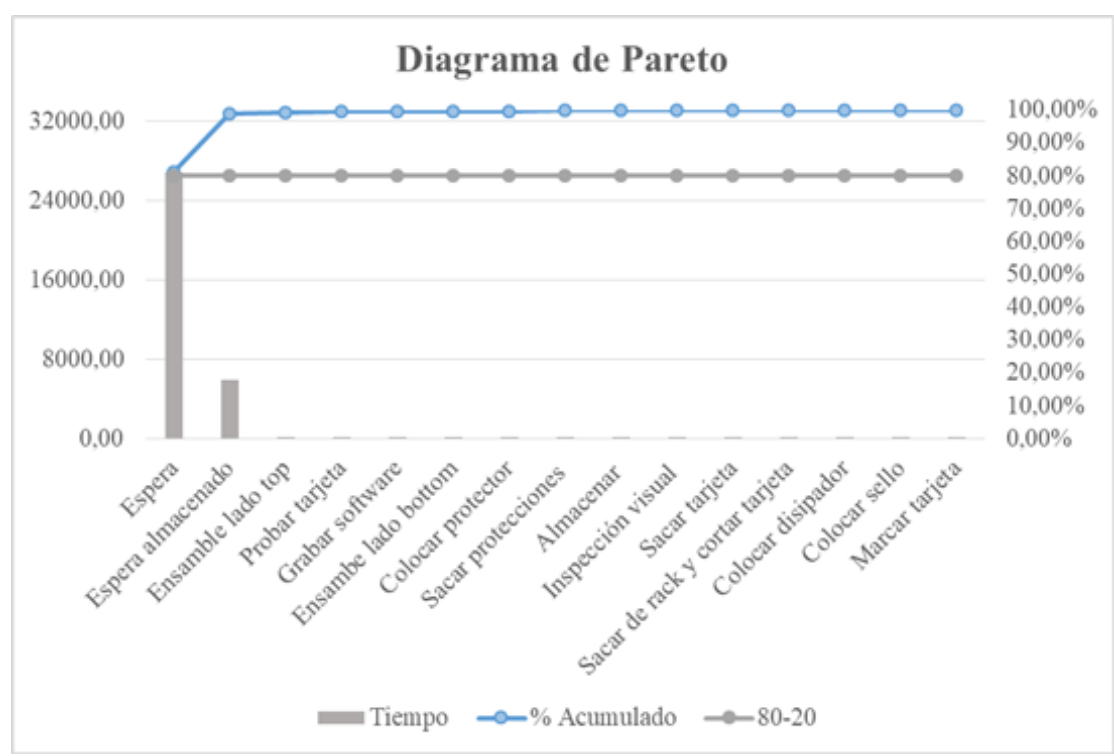

GRÁFICO 6.

Diagrama de Pareto empresa de producción semiautomatizada de tarjetas electrónicas

Elaboración propia a partir de las fichas de levantamiento de procesos

para la empresa de producción semiautomatizada de tarjetas electrónicas.

En lo que se refiere a la empresa de producción manual de motocicletas, al realizar un análisis de todos los modelos, se determinó que el X1 es el que ocupa un mayor tiempo de ensamble, esto se debe principalmente a que el modelo tiene más componentes de materia prima y por ende, la colocación y ensamble de las piezas en el chasis toma mayor duración. A continuación en la Tabla 5 y en el Gráfico 7, correspondientes al diagrama de Pareto, se muestra que la actividad crítica es el ensamble de partes.

TABLA 5.

Análisis estadístico del proceso de ensamblaje del modelo de motocicleta X1

\begin{tabular}{|c|c|c|c|}
\hline \multicolumn{4}{|c|}{ Modelo de motocicleta X1 } \\
\hline Actividades & Tiempo & $\begin{array}{l}\% \\
\text { Acumulado }\end{array}$ & $\begin{array}{l}\text { Tiempo } \\
\text { Acumulado }\end{array}$ \\
\hline $\begin{array}{l}\text { Ensamble de } \\
\text { partes }\end{array}$ & 2866,066 & $37,47 \%$ & 5068,69 \\
\hline $\begin{array}{l}\text { Control de } \\
\text { calidad }\end{array}$ & 2202,628 & $53,75 \%$ & 7271,32 \\
\hline $\begin{array}{l}\text { Armado de } \\
\text { partes }\end{array}$ & 1936,08 & $68,06 \%$ & 9207,40 \\
\hline $\begin{array}{l}\text { Desempaque y } \\
\text { clasificación } \\
\text { de piezas }\end{array}$ & 1809,104 & $81,43 \%$ & 11016,51 \\
\hline $\begin{array}{l}\text { Conectado de } \\
\text { partes }\end{array}$ & 771,54 & $87,13 \%$ & 11788,05 \\
\hline $\begin{array}{l}\text { Tapizado de } \\
\text { monturas }\end{array}$ & 667,266 & $92,06 \%$ & 12455,31 \\
\hline $\begin{array}{l}\text { Preparado de } \\
\text { materiales }\end{array}$ & 422,97 & $95,19 \%$ & 12878,28 \\
\hline Embalaje & 350,749 & $97,78 \%$ & 13229,03 \\
\hline Transporte & 159,834 & $98,96 \%$ & 13388,87 \\
\hline Lubricación & 44,468 & $99,29 \%$ & 13433,33 \\
\hline Calibración & 40,542 & $99,59 \%$ & 13473,88 \\
\hline $\begin{array}{l}\text { Registro de } \\
\text { motocicletas }\end{array}$ & 38,038 & $99,87 \%$ & 13511,91 \\
\hline Levantamiento & 17,074 & $100,00 \%$ & 13528,99 \\
\hline
\end{tabular}


Elaboración propia a partir de las fichas y diagramas de levantamiento de procesos para empresa de producción manual de motocicletas.

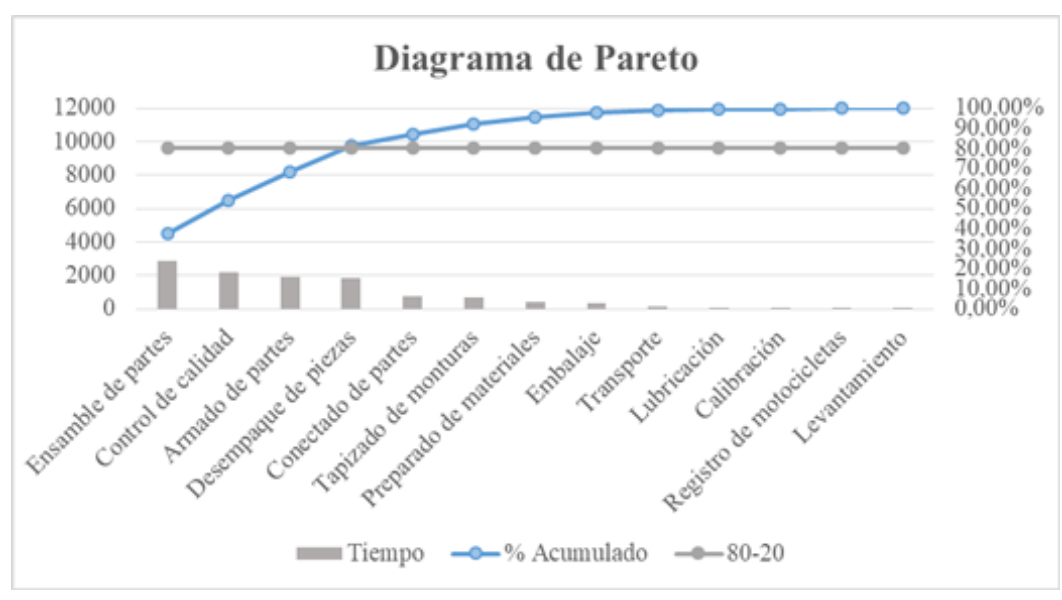

GRÁFICO 7.

Diagrama de Pareto empresa de producción manual de motocicletas

Elaboración propia a partir de las fichas y diagramas de levantamiento

de procesos para empresa de producción manual de motocicletas.

\subsubsection{Diagrama de Cajas o Boxplot}

El Gráfico 8 ilustra que la mayoría de los procesos de ensamblaje de tarjetas electrónicas tienen un tiempo de duración de pocos segundos. Sin embargo, también existen muchos datos atípicos, provocados principalmente por las esperas existentes en algunos procesos.

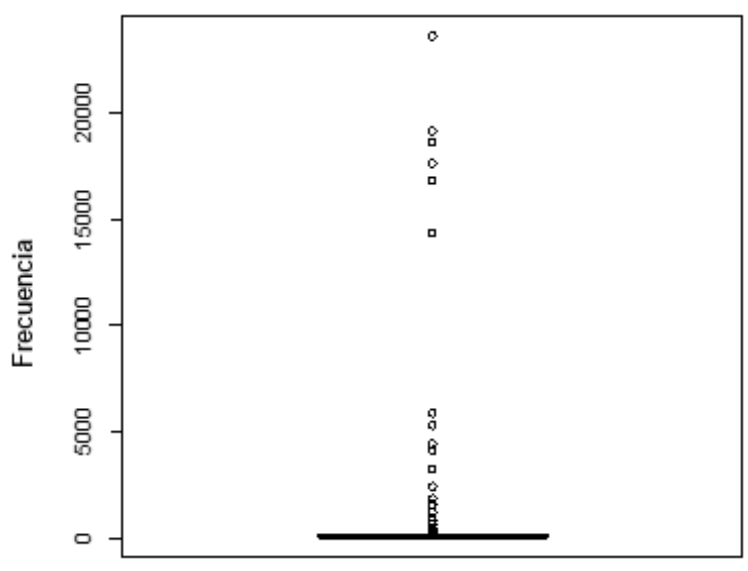

GRÁFICO 8.

Análisis de los procesos de ensamble de la empresa de producción semiautomatizada de tarjetas electrónicas

Elaboración propia a partir de las fichas levantamiento de procesos para la empresa de producción semiautomatizada de tarjetas electrónica.

Por otro lado, con respecto al ensamblaje de motocicletas, como se muestra en el Gráfico 9, los procesos de ensamble tienden a la baja, debido a que una gran cantidad de actividades tiene un tiempo corto de duración; sin embargo, también se pueden visualizar subprocesos atípicos que se concentran en el desempaque y clasificación de piezas, control de calidad, tapizado de monturas, armado de partes y embalaje. Estos subprocesos son ejecutados en un tiempo mayor en relación a los demás. 


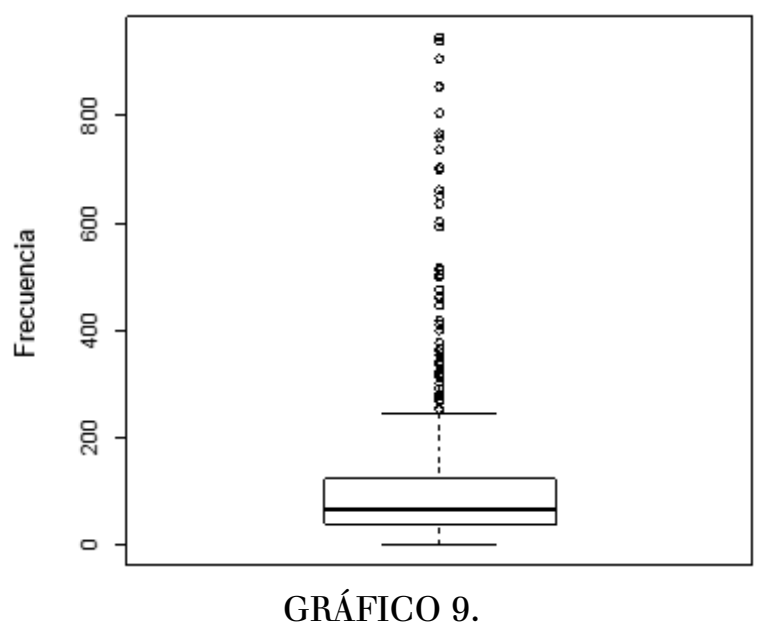

Análisis de los procesos de ensamble de la empresa de producción manual de motocicletas Elaboración propia a partir de las fichas de levantamiento de procesos para empresa de producción manual de motocicletas

\subsubsection{Histograma}

En el Gráfico 10 se puede determinar que la mayor parte de los procesos se encuentran en el intervalo de 0 a 5000 segundos, tendiendo principalmente a cero, lo cual significa que el tiempo de duración de las actividades en el ensamble de tarjetas electrónicas es de pocos segundos.

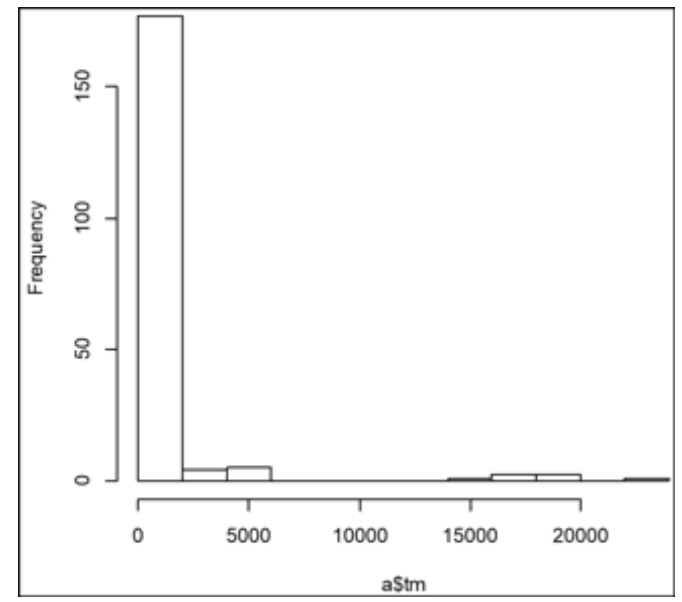

GRÁFICO 10.

Histograma de los procesos de ensamble de la empresa de producción semiautomatizada de tarjetas electrónicas

Elaboración propia a partir de las fichas levantamiento de procesos para la empresa de producción semiautomatizada de tarjetas electrónica.

Con respecto a la línea de ensamblaje de motocicletas, el histograma presentado en el Gráfico 11 muestra que la mayor acumulación de actividades se encuentra en el intervalo de 0 a 200 segundos, lo cual significa que tiende a tener un gran número de actividades cortas en los procesos de ensamble. 


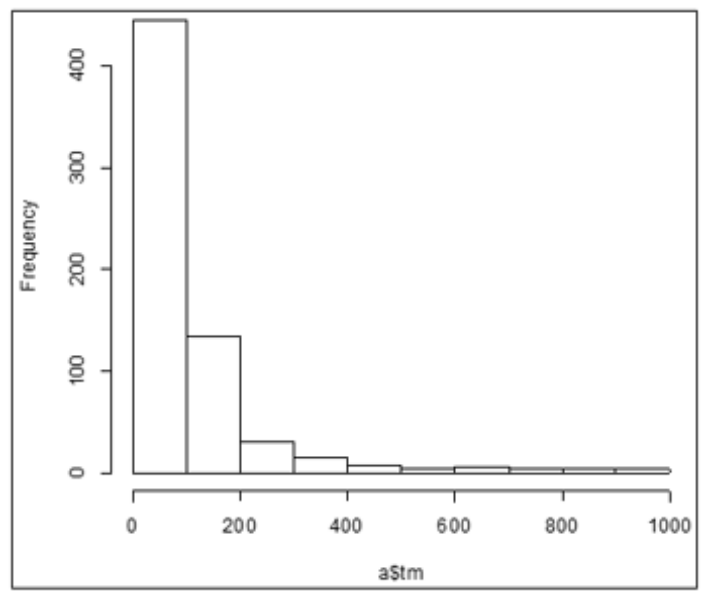

GRÁFICO 11.

Histograma de los procesos de ensamble de la empresa de producción manual de motocicletas Elaboración propia a partir de las fichas levantamiento de procesos para la empresa de producción manual de motocicletas.

Finalmente, al aplicar la metodología para levantamiento de procesos mediante la utilización de fichas y elaboración de diagramas, y con los análisis realizados posteriormente con dicha información, se logró identificar todos los procesos (estratégicos, operacionales y de apoyo) y tiempo necesario para cada uno de ellos. Por lo tanto, se cuenta con información confiable y precisa, que será el pilar fundamental para realizar un correcto análisis con respecto a costos, permitiendo así, establecer el sistema de costeo TDABC y a la vez lograr el desarrollo de un modelo de gestión de procesos eficiente y eficaz.

\section{Discusión}

Es conocido que el levantamiento y análisis de procesos puede ser utilizado como punto inicial para la aplicación de un sistema de costos basado en actividades. Esto se debe a que la identificación de grupos de recursos que intervienen en la ejecución de los procesos de las empresas, en la primera etapa del sistema TDABC, usa como uno de sus parámetros una estimación de las unidades de tiempo requeridas para realizar un proceso, actividad y un servicio. Por tanto, es indispensable reconocer claramente todas las actividades y procesos ejecutados y su importancia para el sistema de costeo TDABC.

Como resultado del levantamiento de procesos en las empresas casos de estudio, se pudo identificar los procesos, subprocesos y las actividades realizadas con los mismos recursos, con el fin de poder agruparlas y así lograr determinar el tiempo necesario para cada proceso de acuerdo a sus características, así como el número de repeticiones correspondiente a las actividades.

Al momento de levantar y procesar información contable, se puede realizar un análisis profundo de los costos referentes a todos los procesos, estableciendo así un sistema de costeo apropiado.

Así también, debido al uso de la metodología propuesta, en la presente investigación se logró identificar los procesos críticos en las empresas estudiadas, los cuales pueden ser objeto de un estudio más profundo que conlleve a la mejora del tiempo invertido en dichas actividades.

\section{Conclusiones}

El levantamiento de procesos y su metodología tienen un carácter integral, puesto que permiten abarcar una gran cantidad de información de las empresas, englobando factores fundamentales. 
Se realizó una clara identificación de cada uno de los procesos, subprocesos y actividades, tanto del área administrativa como del área operativa para conocer procesos críticos y se realizaron validaciones para corroborar la veracidad de la información obtenida y realizar análisis posteriores.

Durante el desarrollo de la investigación fue notoria la aplicación de herramientas estadísticas para analizar la información levantada. Tanto en el Diagrama de Pareto como en el Diagrama de Cajas y en el Histograma, se pudo observar la existencia de dispersión de los tiempos que se utilizan en algunas actividades y por ende, se identificó que los procesos críticos para las dos empresas se encuentran principalmente en el área de ensamble.

En la empresa de producción semiautomatizada de tarjetas electrónicas, al analizar los datos, se concluye que el proceso crítico es el ensamble de modelos automáticos, por lo que se determinó que las esperas que existen entre algunas actividades son las que representan la mayor parte del tiempo total de duración del proceso. Mientras que para la empresa de producción manual de motocicletas, los procesos críticos se sitúan en un gran porcentaje en subprocesos que requieren de mayor tiempo de ejecución (desempaque y clasificación de piezas, control de calidad, tapizado de monturas, armado de partes y embalaje) en comparación a los demás subprocesos, esto debido a que el mayor trabajo en la producción de motocicletas está en la colocación y ensamble de sus piezas en el chasis, la revisión y la preparación final en cada una de ellas.

Otro aspecto importante que se pudo obtener del análisis y levantamiento de información, es determinar que los datos levantados en fichas, presentados en la Tabla 3, así como el mapeo y diagramación de los procesos y actividades sirven como insumo fundamental para llevar a cabo el primer paso de la metodología de costeo TDABC. De esta manera, el presente trabajo representa un aporte importante para la realización de futuras investigaciones; puesto que se convierte en el punto de partida para la integración de los procesos y actividades, en la aplicación del sistema de costeo descrito.

Algunos inconvenientes que surgieron como limitación al obtener información se debieron a que el personal de los departamentos no podía distinguir los tiempos que les toma ejecutar los procesos en cada empresa, puesto que realizaban actividades para otras empresas que forman parte de la misma Corporación. Por todo lo anterior, se pudo establecer que las empresas caso de estudio no laboran de forma independiente debido a que comparten personal del área administrativa.

Finalmente, esta investigación constituye una contribución significativa al aportar con la metodología planteada para el levantamiento de procesos que puede servir en otros tipos de empresas industriales, puesto que la guía metodológica propuesta cuenta con una base teórica y práctica bastante completa, debido a que se han llevado a cabo investigaciones profundas sobre el tema para así, contribuir al conocimiento existente. Además, mediante el levantamiento de procesos desarrollado, se pretende que las empresas logren comprender de mejor manera los procesos que realizan y priorizarlos, con el fin de definir la cadena de valor de la organización, optimizando recursos e incrementando la productividad.

\section{Agradecimientos}

El trabajo presentado forma parte del proyecto de investigación "Modelo de Gestión para la Optimización de Procesos y Costos en la Industria de Ensamblaje”, ganador del XV Concurso de Proyectos de Investigación, financiado por la Dirección de Investigación de la Universidad de Cuenca (DIUC). Se agradece al equipo IMAGINE (Industrial MAnaGement and INnovation $\mathrm{rEsearch}$ ), quienes con su experiencia, conocimiento y motivación hicieron posible el desarrollo de la investigación y a todos los directivos y el personal en general de las empresas casos de estudio por todo el tiempo e información brindada amablemente.

\section{REFERENCIAS BIBLIOGRÁFICAS}

Agudelo Tobón, L. F., \& Escobar Bolívar, J. (2008). Gestión por procesos. Icontec.

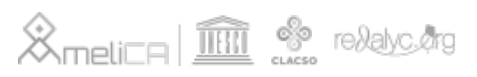


Oakland, J. S. (1989). Total Quality Management: The route to improving performance. Oxford: A ButterworthHeinemann.

Bravo Carrasco, J. (2009). Gestión de Procesos (Con Responsabilidad Social). Santiago de Chile: Evolución S.A.

Beltrán Sanz, J., Carmona Calvo, M. A., Carrasco Pérez, R., Rivas Zapata, M. A., \& Tejedor Panchon, F. (2009). Guia para una gestión basada en procesos. España: Instituto Andaluz de Tecnología.

Franklin Fincowsky, E. B. (2009). Organización de Empresas (Tercera). México: Mc Graw Hill.

International Organization for Standardization. (2015). Norma Internacional ISO 9000:2015. Sistemas de gestión de la calidad: Fundamentos y vocabulario. Recuperado de https://www.iso.org/obp/ui/\#iso:std:iso:9000:ed-4:v1:es

Hitpass, B. (2017). BPM Business Process Management: Fundamentos y conceptos de implementación. Chile.

Ortega Vindas, J. (2009). Guía para el levantamiento de procesos. Ministerio de Planificación Nacional y Política Económica.

Servicio de Evaluación, Planificación y Calidad. (2011). Manual de Gestión de Procesos.

Área de Planificación y Evaluación MOPT Seguridad Vial. (2015, octubre 30). Guía para levantamiento de procesos institucionales y diseño de indicadores.

International Organization for Standardization. (2000a). Norma Internacional ISO 9000:2000. Sistema de Gestión de la Calidad - Conceptos y Vocabulario.

Servicio de Salud de Castilla-La Mancha. (2002). La Gestión por Procesos. Toledo, España: Servicio de Calidad de la Atención Sanitaria.

Instituto Centroamericano de Administración Pública - ICAP. (2006). Análisis de Procesos y Elaboración de Manuales de Procedimiento, MIDEPLAN. San José, Costa Rica.

European Foundation for Quality Management. (1988). Modelo EFQM. Recuperado de http://www.efqm.org/

International Organization for Standardization. (2000b). Norma ISO 9001:2000.

Universidad Politécnica de Valencia. (2006). Plan Pegasus. Recuperado de http://www.upv.es/contenidos/PEGASU S/infoweb/pegasus/info/indexnormalc.html

Asociación Española de Normalización (2008). UNE 93200. Calidad en Cartas de Servicios. España.

Kaplan, R. S., \& Norton, D. P. (1997). El Cuadro de Mando Integral. Gestión 2000.

Porter, M. E. (2000). Estrategia Competitiva. Continental.

Instituto de Normas Técnicas de Costa Rica. (2004). Norma técnica INTECO "INTE-N544 Orientación sobre el concepto y uso del Enfoque Basado en Procesos para los sistemas de gestión. Recuperado de https://www.inteco.org/

Kaplan, R. S., \& Anderson, S. R. (2007). Time-Driven Activity-Based Costing: A simpler and more powerful TimeDriven Activity-Based Costing: A simpler and more powerful path to higher profits. Boston, MA, USA: Harvard Business School Press.

Arbulo Lopez, P. R., \& Fortuny-Santos, J. (2011, abril). Innovación en gestión de costes: del ABC al TDABC. Dirección y Organización, 43, 11.

Astudillo Delgado, V. H., \& González Martínez, D. E. (2014). Propuesta de Modelo de Gestión por Procesos en la Ensambladora de Motocicletas CKD de la empresa Vycast Cía. Ltda., bajo lineamientos ISO 9001. (Universidad del Azuay). Recuperado de http://dspace.uazuay.edu.ec/handle/datos/5019

Orbe Aguirre, H. D. (2018). Plan de Negocio para la Creación del Área de Ensamblaje de Televisores aplicado a la Empresa Ecuatoriana Ensab para la Comercialización a Nivel Nacional. (Universidad Internacional del Ecuador). Recuperado de http://repositorio.uide.edu.ec:8080/xmlui/handle/37000/2535?show=full

Criollo Coronado, L. A. (2016). Plan de Negocios para la Producción Nacional y Ensamblaje de Bicicletas Eléctricas con Componentes Nacionales (Universidad Internacional del Ecuador). Recuperado de https://repositorio.uide.edu .ec/handle/37000/1574

Guerrero, P., Guamán, R., Colina Morles, E., \& Siguenza-Guzmán, L. (2019). Modelo de Optimización para el cálculo de tiempos en procesos de ensamblaje. 
Erika X. Benavídez Vera, et al. LEVANTAMiento de Procesos COMO baSE Para La APLICACIÓN DE SISTEMAS...

Ramírez, J., \& Guamán, R. (2019). Predicción de tiempos estándar en líneas de ensamble usando minimos cuadrados en modelos lineales multivariables.

Rodas D., P., Colina Morles, E., Peña, M., \& Siguenza-Guzmán, L. (2019). Modelo matemático basado en programación lineal y lógica difusa para predicción de tiempos en industrias de ensamble de bicicletas. Revista RISTI.

Luzuriaga, A. (2019). Modelo Genérico de Procesos para empresas de ensamblaje.

García Criollo, R. (2005). Estudio del trabajo: Ingeniería de métodos y medición del trabajo (Segunda). México: Mc Graw Hill.

\section{Notas}

[1] https://www.bizagi.com/es/productos/bpm-suite/modeler

(C) Universidad de Cuenca 2019

CC BY-NC-SA

INFORMACIÓN ADICIONAL

Código JEL:: M11, M41. 